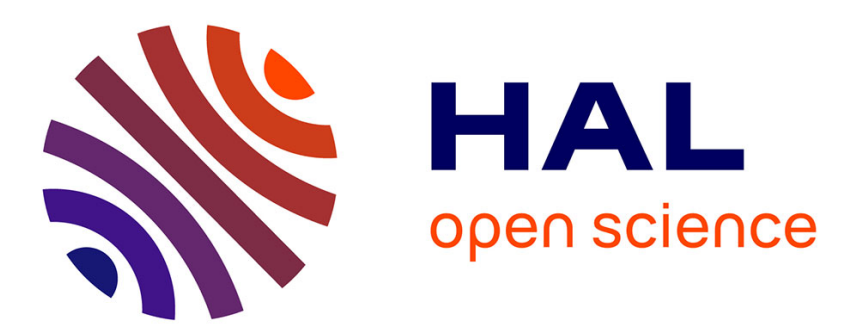

\title{
Domain decomposition for coupling finite and boundary element methods in EEG
}

\author{
Emmanuel Olivi, Maureen Clerc, Théodore Papadopoulo
}

\section{To cite this version:}

Emmanuel Olivi, Maureen Clerc, Théodore Papadopoulo. Domain decomposition for coupling finite and boundary element methods in EEG. Biomag 2010, S. Supek, Apr 2010, Dubrovnik, Croatia. pp.120-123, 10.1007/978-3-642-12197-5_24. inria-00497099

\section{HAL Id: inria-00497099 \\ https://inria.hal.science/inria-00497099}

Submitted on 2 Jul 2010

HAL is a multi-disciplinary open access archive for the deposit and dissemination of scientific research documents, whether they are published or not. The documents may come from teaching and research institutions in France or abroad, or from public or private research centers.
L'archive ouverte pluridisciplinaire HAL, est destinée au dépôt et à la diffusion de documents scientifiques de niveau recherche, publiés ou non, émanant des établissements d'enseignement et de recherche français ou étrangers, des laboratoires publics ou privés. 


\title{
Domain decomposition for coupling finite and boundary element methods in EEG
}

\author{
Emmanuel Olivi ${ }^{1}$, Maureen Clerc $^{1}$ and Théodore Papadopoulo ${ }^{1}$ \\ ${ }^{1}$ INRIA Odyssée Project-Team, Sophia Antipolis, France
}

\begin{abstract}
The forward problem in electroencephalography aims to simulate on the scalp the potential $V$ of an electromagnetic field generated by a simulated source. It must fit precisely with the electromagnetic propagation in the patient head. Yet, the skull anisotropy happens to be highly anisotropic, and must then be modeled. Although boundary element methods cannot deal with anisotropy like finite element methods, the symmetric BEM offers a higher accuracy than FEM wherever the conductivity can be considered as constant (i.e. for the brain and the scalp). A domain decomposition (DD) framework allows to split the global system into several ones with smaller computational domains. Then, one method (BEM or FEM) can be used per volume. This work presents such a coupling formulation of a 3-DD method solving iteratively a BEM for the brain, a FEM for the skull layer, and finally a BEM for the scalp.
\end{abstract}

Keywords- EEG, BEM, FEM, Coupling, Domain Decomposition

\section{INTRODUCTION}

The problem of finding the sources in the brain that are responsible for a measured EEG signal is an inverse problem of localization whose resolution requires many resolutions of the forward problem. The latter computes the potential $V$ on the scalp of an electromagnetic field generated by a simulated source (in our case a dipole $J_{p}$ ). This propagation through the head is governed by the Maxwell equations in their quasi-static approximation. The forward problem resolution is achieved solving for $V$ the Poisson equation:

$$
\begin{cases}\nabla \cdot(\Sigma \nabla V)=\nabla \cdot J_{p} & \text { in } \Omega \\ (\Sigma \nabla V) \cdot n=0 & \text { on } \Gamma\end{cases}
$$

,with $\Sigma$ the head tissues' conductivity and $\Gamma$ the Scalp/Air interface (outflow current through the neck is neglected). The forward model must fit precisely with the electromagnetic propagation in the patient head. Although the white matter makes the brain anisotropic, the skull anisotropy has a strong influence on the results, and must therefore be taken into account. Indeed, the skull conducts electricity ten to eighty times stronger in tangential directions than in the ra- dial one. Finite Element Methods (FEM:s) can deal with this anisotropy, but offer a poor accuracy when compared to the symmetric Boundary Element Method (sBEM) in homogenous media. Therefore the coupling of both should yield better accuracy ; using the BEM wherever the conductivity can be considered as constant (i.e. for the scalp and the brain when we neglect white matter anisotropy), and the FEM for the skull.

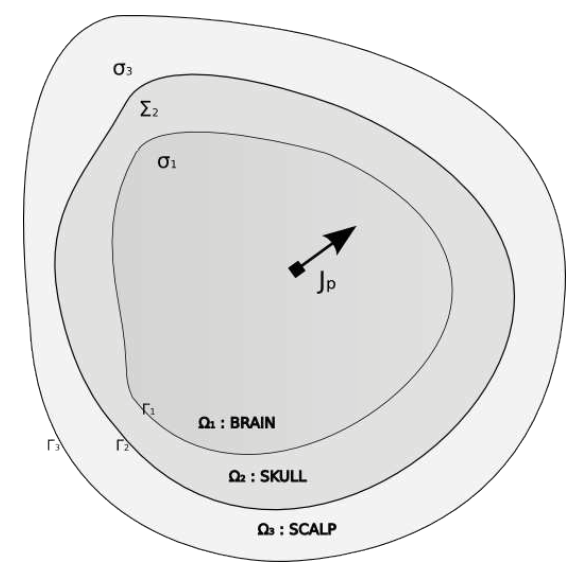

Fig. 1: A three-layer head model with nested regions.

\section{THE SYMMETRIC BEM, THE IMPLICIT FEM AND COUPLING FORMULATIONS}

\section{A. The symmetric BEM, and the implicit FEM}

Wherever conductivity is considered as constant in a set of nested regions (see Fig. 1) then the first equation in Eq. 1 becomes $\sigma \Delta V=\nabla \cdot J_{p}$ and can be solved using integral formulations. Instead of the classical formulation that involves double-layer potentials, we use a formulation that involves both double-layer and single-layer potentials, to yield a symmetric system of equations. The symmetric BEM [1] involves both the potential and the normal current as unknowns on triangulated surfaces describing the different regions.

FEM:s usually require to mesh the entire volume to spread the information from the source through the computational 
domain. The geometry used to solve the forward problem in EEG comes from anatomical MRI:s. These 3D-images are then segmented to obtain a levelset description of the regions to model. Generation of the (most often tetrahedral) meshes can be achieved but is usually expensive computationally and time consuming. The implicit FEM [3] bypasses this mesh generation step, going directly from the levelsets of the interfaces separating the various domains to the matrix associated to the FEM. It works on Cartesian grids with the potential V at the node locations as unknown, described with Q1 finite elements.

The sBEM developped in the open-source code OpenMEEG, turns out to be much more accurate than other BEM:s, and also than the implicit FEM (iFEM).

\section{B. A BEM-FEM coupling}

Domain decomposition is a widely used numerical technique that allows to split the computational domain into regions of constant conductivities on the one hand and the skull on the other hand. We will then be able to use the appropriate method (BEM or FEM) per volume. Boundary conditions ensure the communication between the sub-problems. Several iterations are needed to solve the global system, and a relaxation at the interfaces is compulsory to ensure convergence.

We first propose a Neumann-Dirichlet approach which iteratively solves a BEM for the brain region, and a FEM for the skull and the scalp region. At iteration $k$, we first solve a BEM with Neumann boundary condition, to obtain $V$ at the brain/skull interface. Secondly, a mixed Dirichlet - homogeneous Neumann problem is solved with the FEM using the newly computed $V$ at the brain/skull interface as boundary condition. This gives $V$ on the scalp:

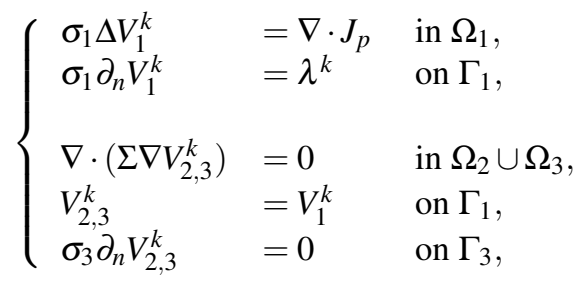

Finally, $\lambda^{k+1}$ is updated by combining the normal current computed by the FEM on the brain/skull interface and the previous $\lambda^{k}$ with a relaxation parameter $\omega$ :

$$
\lambda^{k+1}=(1-\omega) \lambda^{k}+\omega\left(\Sigma_{2} \nabla V_{2}^{k}\right) \cdot n \text { on } \Gamma_{1}, \omega>0
$$

We also present a Neumann-Dirichlet-Neumann coupling procedure, restricting the FEM to the skull only (see Fig. 2), and completing the computations with a BEM for the scalp:

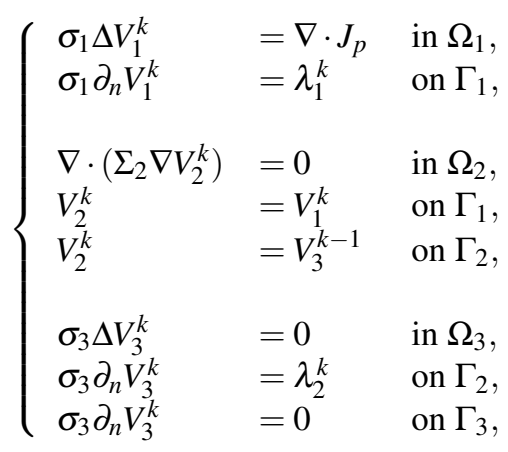

With relaxations made on the Neumann's parameters $\lambda_{1}$ and $\lambda_{2}$ :

$$
\begin{array}{ll}
\lambda_{1}^{k+1}=\left(1-\omega_{1}\right) \lambda_{1}^{k}+\omega_{1}\left(\Sigma_{2} \nabla V_{2}^{k}\right) \cdot n & \text { on } \Gamma_{1}, \omega_{1}>0 \\
\lambda_{2}^{k+1}=\left(1-\omega_{2}\right) \lambda_{2}^{k}+\omega_{2}\left(\Sigma_{2} \nabla V_{2}^{k}\right) \cdot n & \text { on } \Gamma_{2}, \omega_{2}>0
\end{array}
$$

Initial condition for this coupling scheme is: $V_{3}^{0}=0$, with the relaxation parameters: $\omega_{1}=\omega_{2}=1$ for $k=1$. For $k>1$, relaxation parameters are set manually to $\omega_{1}=\omega_{2}=0.7$; a higher relaxation parameter would make the scheme diverge.

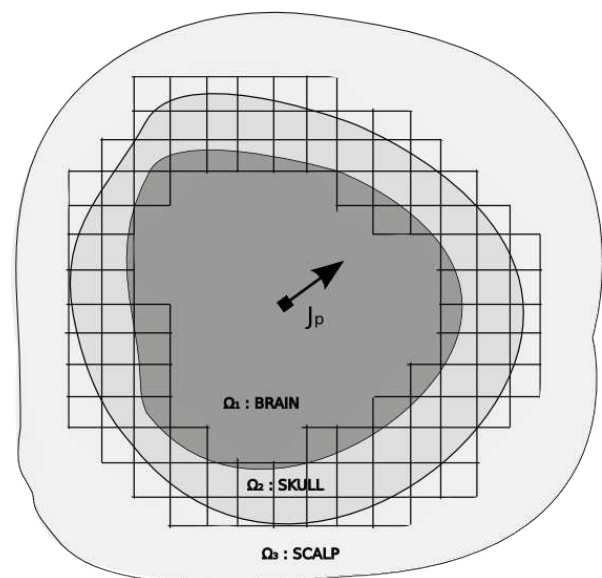

Fig. 2: The three-layer head model with the FEM's grid for the skull.

Special care must be observed for the Neumann problem, indeed no accumulation of current should appear, therefore numerically we ensure that:

$$
\begin{array}{ccc}
\int_{\Omega_{1}} \nabla \cdot J_{p}+\int_{\Gamma_{1}} \lambda_{1}^{k} & =0 & , \forall k \\
\int_{\Gamma_{2}} \lambda_{2}^{k} & =0 & , \forall k
\end{array}
$$




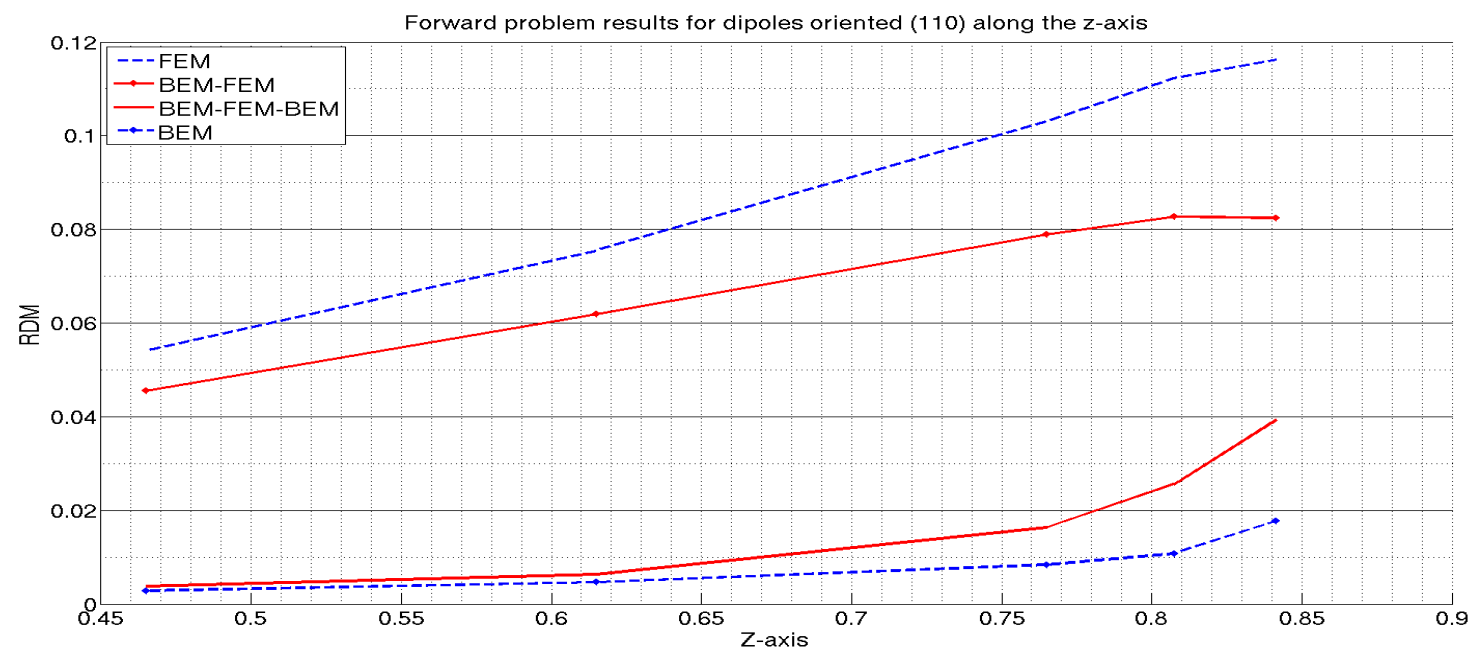

Fig. 3: RDM for the isotropic case, for pure and coupled methods.

\section{NUMERICAL RESULTS.}

In order to validate results of the forward problem, analytical solutions are computed on a three-layer concentric sphere model [2], for both isotropic and anisotropic skull layer. Radii of the spheres and conductivities of the different layers are respectively $\{0.87,0.92,1.0\}$ and $\{1.0,0.0125,1.0\}$ for the isotropic case. Considering the anisotropic one, we have set up the conductivity in the tangential direction to ten times the normal one. The sBEM is computed on 642 point mesh per surface, and the iFEM considers a Cartesian grid of 90 points in each direction. The BEM-FEM coupling as well as the BEM-FEM-BEM coupling use the same previous grid sizes. Computations have been done for 5 dipoles oriented in Cartesian coordinates: $(1,1,0)$, and locations along the Z-axis: $\{0.465,0.615,0.765,0.8075,0.8415\}$. Accuracies of the numerical solutions are given by the Relative Difference Measure (RDM) of the potential on the scalp:

$$
R D M=\left\|\frac{V_{\text {analytic }}}{\left\|V_{\text {analytic }}\right\|}-\frac{V_{\text {computed }}}{\left\|V_{\text {computed }}\right\|}\right\|
$$

Fig. 3 shows the RDM for both pure and coupled methods in the isotropic case. The iFEM method in blue (dashed) is the less precise due to the poor grid definition. We see that the sBEM is the most accurate with a RDM always below the $2 \%$. The BEM-FEM coupling in red (full with diamonds) shows a slight improvement compared to the accuracy of the FEM, whereas the BEM-FEM-BEM coupling really inherits its accuracy from the BEM. Coupling process have been stopped at
40 iterations. At this point the relative residuals computed as: $\frac{\left\|\left.\left(\Sigma_{2} \nabla V_{2}^{k+1}\right) \cdot n\right|_{\Gamma_{i}}-\lambda_{i}^{k}\right\|}{\left\|\lambda_{i}^{k}\right\|}$, for $i=1,2$ were all below $6.10^{-5}$. These results do not show the full benefit of the coupling, because the coupling takes its sense for anisotropic conductivities. As BEM cannot handle anisotropy, they must model the skull as isotropic and therefore their high accuracy is partly wasted because of the coarse model approximation. On Fig. 4, we have plotted the result of the BEM with an isotropic skull whose conductivity equals 0.0125 compared with the anisotropic analytical solution with conductivities $($ radial, tangential $)=(0.0125,0.125)$. The iFEM as well as the BEM-FEM-BEM coupling have been computed with an anisotropic skull. This time, results have been compared on 15 dipoles: the five previous locations for three different orientations: $(1,0,1)$ in blue, $(1,1,0)$ in green and $(0,0,1)$ in red color. One can see that the coupling (full line) has a better precision than the BEM (dashed line with diamonds) for all dipoles expected the two located at $(0,0,0.8415)$ with orientations $(1,0,1)$ and $(0,0,1)$. As it comes closer to the skull, the singularity of the dipole makes the approximation due to the iFEM's grid worse. Using a higher resolution FEM's grid, this error should go diminishing. Results on the blue curve for the coupling are not gratifying; there are some convergence problems with our BEM-FEM-BEM algorithm, for some dipole orientations, that we have not yet solved. 


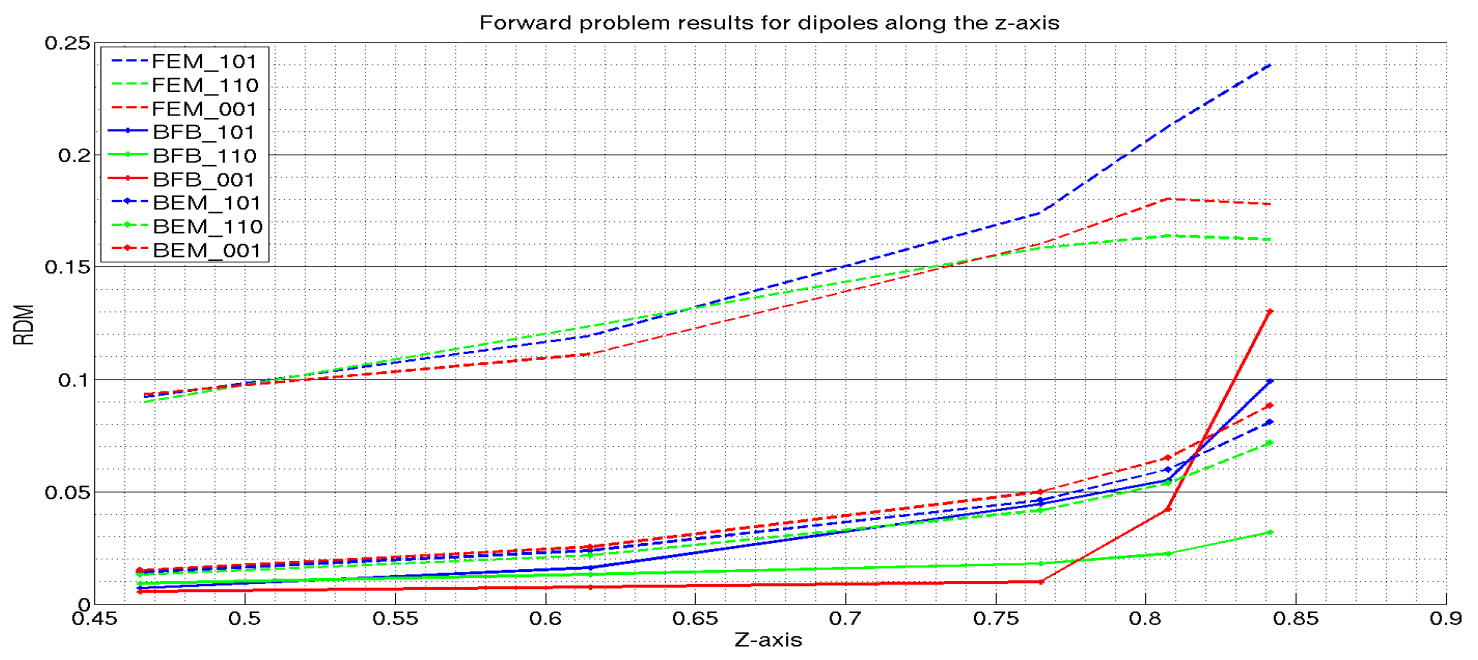

Fig. 4: RDM for the anisotropic case, for pure and coupled methods.

\section{CONCLUSION}

The domain decomposition framework really allows to take advantage of both the versatility of the FEM in modeling conductivity and the accuracy of the BEM. It gives hope for providing accurate solutions of the forward problem without resorting to diminishing the mesh size in finite element methods. One could try to find a relaxation parameter changing through iterations to faster convergence. Parallelism is often used in the domain decomposition community, but it was not pertinent in our case since the sBEM has a direct solver, and only requires a matrix vector product to solve a new Neumann problem for each iteration; most of the time is spent in the iterative solver of the FEM.

\section{REFERENCES}

1. J. Kybic, M. Clerc, T. Abboud, O. Faugeras, R. Keriven, and T. Papadopoulo. A common formalism for the integral formulations of the forward EEG problem. IEEE Transactions on Medical Imaging, 24:1228 , jan 2005.

2. Zhi Zhang A fast method to compute surface potentials generated by dipoles within multilayer anisotropic spheres Phys. Med. Biol. 1995

3. Théodore Papadopoulo and Sylvain Vallaghé. Implicit meshing for finite element methods using levelsets. In Proceedings of MMBIA 07, 2007.

Author: Emmanuel Olivi Institute: INRIA

Street: 2004 route des lucioles BP93

City: Sophia-Antipolis Cedex

Country: France

Email: Emmanuel.Olivi@sophia.inria.fr 